\title{
Fault Detection and Isolation Based on Bond Graph Models: Application to an Electromechanical Actuator
}

\author{
Gabriel dos Santos Sobral and Luiz Carlos Sandoval Góes \\ Department of Mechanical Engineering, Instituto Tecnológico de Aeronáutica (ITA), São José dos Campos, SP/Brazil \\ E-mail: sobral.gbrl@gmail.com; goes@ita.br
}

\begin{abstract}
Driven by the rapid development towards the concept of more electric aircraft in recent years, the power-by-wire technology, especially electromechanical actuators (EMA), is being progressively adopted with the promise of bringing performance improvements for future aircraft in different areas such as weight, maintainability and fuel consumption. However, for safetycritical applications like primary flight controls, the use of EMA introduces specific issues related to the actuator's response to failure. To that end, focusing on EMA fault diagnosis, this work proposes the development of a model-based quantitative Fault Detection and Isolation (FDI) methodology based on bond graph models. Firstly, an EMA behavioural model is created in bond graph, considering the actuator's most common failure modes: stator winding faults, backlash, jamming, and mechanical disconnection. Next, this behavioural model is applied to create a Diagnostic Bond Graph (DBG) model, which is based on the use of virtual residual detectors, or residual sinks, for numerical residuals generation, without the need for deriving symbolic Analytical Redundancy Relations (ARRs). These residuals are then evaluated with experimentally defined thresholds for fault detection, indicating if the system behaviour has departed from the acceptable operating range. Finally, the causal and structural properties of the bond graph are used to derive a Fault Signature Matrix (FSM) through the analysis of the DBG model causal paths. The FSM is employed for fault isolation, reducing the number of fault candidates, in order to isolate the component responsible for the faulty behaviour. Simulation results, using bond graph models implemented in the 20-sim environment, show the successful detection and isolation of all EMA modelled faults using the proposed method.
\end{abstract}

Keywords: Bond Graph, Electromechanical Actuator, Fault Detection, Fault Isolation

\section{Introduction}

Although not new in the industry, the more electric aircraft (MEA) concept is receiving a growing interest in recent years, aiming at the development of safer, lower-cost, and greener technologies for the next generation aircraft. One of the main trends towards increasing the use of electrical power in aircraft systems resides in the adoption of power-by-wire (PBW) actuators. The PBW technology seeks the replacement of conventional hydraulic servo actuators (HSA) by electrically powered systems, with a series of advantages due to the exclusion of the heavy and bulky hydraulic system. With the recent engineering progresses in PBW actuation systems, these actuators are already found in service, with electric-hydrostatic actuators (EHA) and electromechanical actuators (EMA) becoming sufficiently mature to be introduced in the latest commercial and military aircraft [1-3]. Among the various kinds of actuators, EMAs are chosen for this study because of their growing role in aerospace applications.

Despite the potential benefits from EMA adoption such as the possible weight reduction, lower fuel consumption, and reduced maintenance costs, EMAs are not considered mature enough to completely replace conventional HSAs in safetycritical functions such as primary flight controls, where safety concerns due to the probability of jamming and backlash mechanical faults prevents the industry to embrace this technology. To that end, targeting the improvement on safety, reliability, availability and maintainability of complex engineering systems such as EMAs, it is crucial to develop autonomous supervisory systems that are able to quickly detect abnormal behaviours and isolate the causes of any fault which could seriously degrade the performance of the system.

EMA fault diagnosis poses an interesting research problem as the system is composed of several interacting components in different physical domains, resulting in intricate failure modes. Modelling a system with such characteristics can be accomplished by the use of the bond graph methodology, which is particularly suitable for modelling multidisciplinary engineering systems, and can also be used for the develop- 
ment of powerful Fault Detection and Isolation (FDI) algorithms. From this perspective, the use of model-based and simulation-driven techniques can unquestionably provide engineers with efficient means to address these critical issues. Therefore, modelling and off-line simulation becomes a focus, not only to support conceptual design but also to the implementation of FDI systems before it is built and introduced in the industry.

The present work aims to develop a model-based quantitative FDI procedure, based on bond graph models, with application to electromechanical actuators. The proposed method will provide product development engineers with new tools to address EMA fault diagnosis, anticipating FDI since the conceptual design of the system. To accomplish that, an EMA bond graph model, representative of the main dynamic effects of the system, will be developed. That model shall also contemplate the most common failure modes of an electromechanical actuator, making it possible to simulate the system behaviour in different faulty scenarios. From this model, a FDI system based on the technique of Diagnostic Bond Graph (DBG) will be designed. The DBG will then be coupled with the behavioural model for off-line simulation, validating the applicability of the proposed method, revealing what faults can be detected and whether they can be isolated or not.

This work is structured as follows: Section 2 provides a review on FDI techniques and details the proposed bond graph model-based quantitative FDI, defining the steps for implementation of the DBG and construction of the Fault Signature Matrix (FSM). Section 3 presents the bond graph modelling of the EMA using an incremental approach, modelling the electric motor, mechanical power transmission and load coupling subsystems, which are connected to create the EMA behavioural model; the main EMA failure modes are also modelled and incorporated to the bond graph model; next, the DBG and FSM are generated according to the proposed method. Section 4 provides the implementation of the bond graph models and the FDI framework in the 20-sim simulation environment, giving results from the actuator model simulation response and the residuals evaluation for EMA failure modes. The results are discussed in Section 5, which also proposes the topics for future research. Finally, Section 6 presents the conclusions of this work.

\section{Fault Detection and Isolation}

Before discussing any diagnostic system details, it is necessary to clearly define what is understood as a fault. In the aeronautical industry a standard definition is given by Aerospace Recommended Practice (ARP) 4761, Guidelines and Methods for Conducting the Safety Assessment Process on Civil Airborne Systems and Equipment, from SAE International [4]:

Fault: an undesired anomaly in an item or system that may lead to a failure.

Failure: a loss of function or a malfunction of a system or a part thereof.
In this work's context, a more precise interpretation is necessary and the fault definition commonly accepted in the academic literature will be used. This definition is given by Venkatasubramanian [5], as follows:

The term fault is generally defined as a departure from an acceptable range of an observed variable or a calculated parameter associated with a process. This defines a fault as a process abnormality or symptom, such as high temperature in a reactor or low product quality and so on.

Once a fault is clearly defined, the diagnostic system can be divided in four distinct phases [6] as follows: fault detection, fault isolation, fault identification, and fault accommodation. This work will focus on FDI algorithms, comprising the two first steps of this process.

The fault detection stage is concerned in determining if the system behaviour has departed from the acceptable range of operation, raising a fault alarm in case of unacceptable behaviour. The second stage, fault isolation, has the objective of reducing the number of fault candidates, using one or more decision procedures to isolate the component responsible for the fault behaviour.

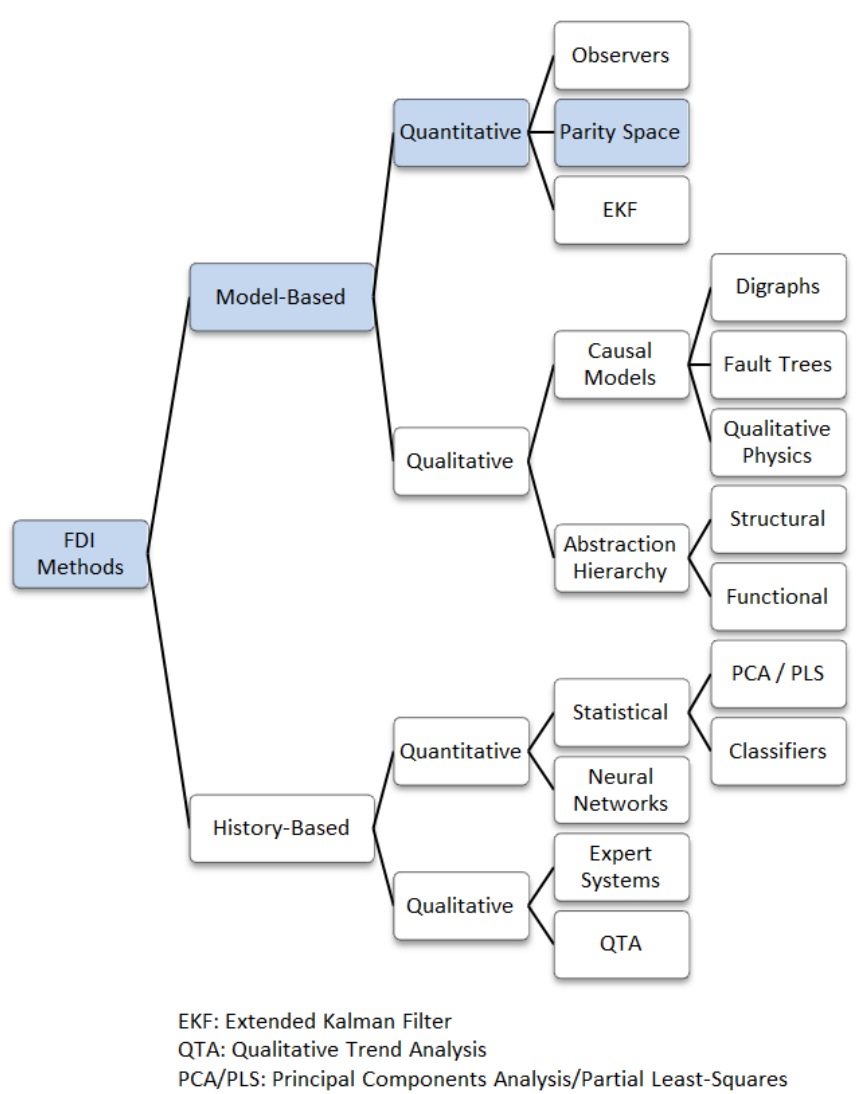

Figure 1: Classification of FDI algorithms. Adapted from [5]

In order to perform these tasks, any FDI algorithm will need $a$ priori knowledge about the system, regarding the set of possible failures and the relationship between the observed variables and these failures, i.e. a set of causal relationships. The 
FDI algorithms may be broadly classified in two classes, depending on how it develops the a priori knowledge about the system. Model-based algorithms derive its knowledge from a fundamental understanding of the physics of the process and its causal relationships. On the other hand, history-based algorithms will rely on past experience with the process, requiring the availability of a large amount of historical data. Regardless of the origin of that knowledge, it can be analysed on a quantitative or qualitative approach. Different FDI methods have been developed in the literature, these methods are presented in fig. 1, following the discussed classification.

This work explores a model-based quantitative FDI method, using the parity space approach. These techniques are based on a comparison of outputs from the real process with those from a behavioural model that describes the non-faulty process, creating redundancy relations. Any discrepancies between the two are described by parity vectors, or residuals. Every residual is expected to be zero when no fault is present and distinguishably different from zero when a fault occurs.

In effect, a perfectly accurate mathematical model of the system is never available. Therefore, there will always be a mismatch between the measured system variables and its mathematical model, even under normal operation, i.e. residuals will always be different from zero, even in the absence of faults. Because of that, it is necessary to establish thresholds for the acceptable levels of each residual. Once the residuals are generated, they are monitored for exceedances over these thresholds, in which case a fault alarm is declared. The subsequent analysis of the residuals leads to fault isolation. The basic scheme for model-based quantitative FDI is given in fig. 2 showing the two basic steps of residual generation and residual evaluation, to be detailed in the following sections.

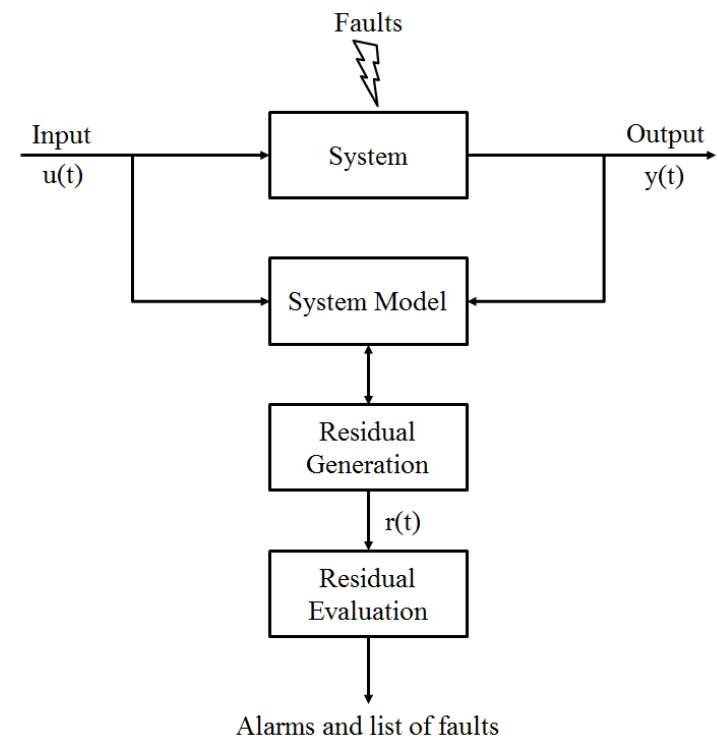

Figure 2: A general scheme for quantitative model-based FDI.

\subsection{Residual Generation: Diagnostic Bond Graph}

A proper residual design method is the best way to achieve the requirements of isolability and robustness on the quantitative model-based FDI. Appropriate residual generation, with robust and structured residuals, will make the residual evaluation step relatively easy.

The bond graph formalism is a knowledge representation form suitable for developing models of multi-energy domain engineering systems, being capable of holding a large amount of structural, functional and behavioural information about the system and describing essential causal relationships. These are reasons that make bond graph models a powerful tool for the construction of model-based FDI systems.

The most common method for generating residuals from bond graph models is through the analytical evaluation of the model physical constraint laws. These constraint laws are written symbolically, eliminating the unknown variables in a way that they will only depend on the system inputs, the parameter values and the measurements from sensors. Such equations are termed Analytical Redundancy Relations (ARR) and the numerical evaluation of ARRs give the residuals. Obtaining ARRs from bond graph models can be a straightforward and structured process, following the causality inversion approach, well documented in the literature [6]. However, a closed symbolic expression cannot be derived for all kinds of processes with all kinds of instrumentation because it may not be possible to, in certain cases, eliminate all unknown variables from the model through symbolic algebra, e.g. when the bond graph model contains algebraic loops that cannot be eliminated.

Samantaray [6] proposes a modified method for direct numerical evaluation of the residuals, introducing the DBG model, with no need for symbolically derivation of ARRs, being applicable to all situations whether the set of constraints can be symbolically resolved or not. The proposed method leads to the same set of residuals which can be obtained through classical means, if the equations are symbolically resolvable. For the construction of the DBG model, preferred differential causality is assigned to the storage elements of the bond graph model, along with the inversion of sensor causalities. This means sensors will impose the measured variable on the system, i.e. sensors become sources.

For instance, given an effort detector, De, the flow in the bond connected to this sensor is equal zero. When the causality is inverted for this sensor it becomes an effort source, imposing the measurement from the real process, and the expression for the source loading, the flow variable, is equated to zero. This expression is indeed, a residual, and its output is measured by a virtual flow sensor, which has only computational existence. All virtual sensors measuring residuals are presented with a superscript '*': Df* or De*. The bond graph for substitution of the inverted causality effort sensor, containing the virtual residual sensor, is presented in fig. 3 .

Similarly, the substitution proposed for the inverted causality flow sensor is dual to the one discussed for the effort sensor. Using this method there will be a virtual residual sensor for 


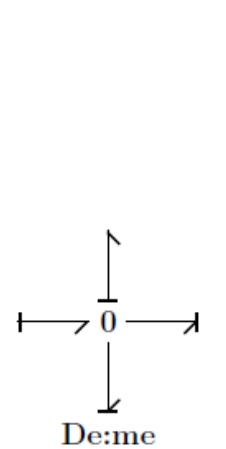

(a)

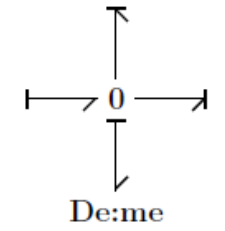

(b)

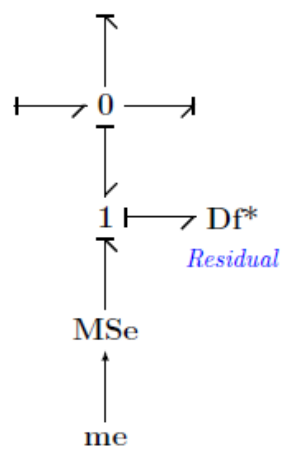

(c)
Figure 3: (a) Effort sensor; (b) Effort sensor in inverted causality; (c) Corresponding substitution and residual virtual sensor. Note: me represents a generic measured effort variable.

each real sensor on the system model. Furthermore, for a sensor in inverted causality, there exists at least one causal path to it which is different or does not exist when compared to the causal paths to any other sensor in the inverted causality. This proves that all the virtual residual sensors have distinct signatures [6]. A similar approach, which refers to these virtual sensors as residual sinks, has been developed by Borutzky [7].

\subsection{Residual Evaluation: Fault Signature Matrix}

Once the residuals are generated, the next step is residual evaluation, which aims to isolate the faulty components in the system's model. The proposed method uses a FSM for that. Each residual is sensitive to some faults and insensitive to some others. For good isolability it is important that different residuals become sensitive to different faults. If a residual fault signature, i.e. the faults to which this residual is sensitive, is different from the fault signatures of any other residuals, the residual is termed structurally independent of the others. The DBG approach guarantees that the number of structurally independent residuals obtained is equal to the number of sensors in the system [6].

The FSM, $S$, will describe the structural sensitivity of each residual to possible faults in the system's model, forming a structure that links the residuals to discrepancies in each component of the system. The elements in the $S$ matrix are determined from the analysis presented in eq. 1.

$$
S_{i j}= \begin{cases}1, & \begin{array}{l}
\text { if the } i \text {-th residual is sensitive to } \\
\text { faults in the } j \text {-th component; }
\end{array} \\
0, & \text { otherwise. }\end{cases}
$$

Additionally, two indexes are assigned to each component on the FSM. The monitorability index $M_{j}$ will be 1 if at least one residual is sensitive to it, in other words, the component's fault is monitorable. The isolability index $I_{j}$ is set to 1 when the component's fault signature is different from fault signatures of all other components, that is, the fault can be isolated. Since the DBG approach does not necessarily have closed form ARRs, the fault signatures are obtained by analysis of the bond graph causal paths to each residual.

Once the residuals are obtained from the DBG model and the FSM is constructed, a decision procedure shall be applied to the set of evaluated residuals in order to generate the alarms. A threshold $\varepsilon_{i}$ has to be defined for each residual $r_{i}$. The necessity for this non-zero threshold bounding the residual is due to process uncertainties, modelling errors and measurement noises.

\section{Application to an Electromechanical Actua- tor}

EMAs can have different configurations, adapted to the requirements of particular operations. As a more general application, the linear EMA architecture is mainly considered and discussed in this study. The linear EMA can have a gearbox or not, in order to reduce the electric motor speed before the power is transmitted to the load. The gear-drive EMA is more susceptible to certain single-point failures that would generate an undesired mechanical jamming, bringing complications to critical applications. On the other hand, direct-drive EMAs are less prone to mechanical jamming, have less probability of actuator damping loss, increased efficiency and reliability, and reduced system inertia due to the elimination of the inbetween gearbox [1]. However, these advantages do not come for free, requiring a high-performance servo motor with high torque density and reliability, and in the absence of the gearbox buffering effect, any load disturbance can be directly reflected to the motor and the control system. For these reasons the direct-drive EMA will be studied in the present work.

The schematic for a direct-drive linear EMA is presented in fig. 4. Electric power is provided by the aircraft electrical buses and delivered to the actuator by the Power Drive Electronics (PDE) according to the command signal from the Actuator Control Electronics (ACE). The electric power is transformed into rotational mechanical power by the Electric Motor (EM), and then, converted into translational mechanical power by a Mechanical Power Transmission (MPT) mechanism, transferring power to the control surface.

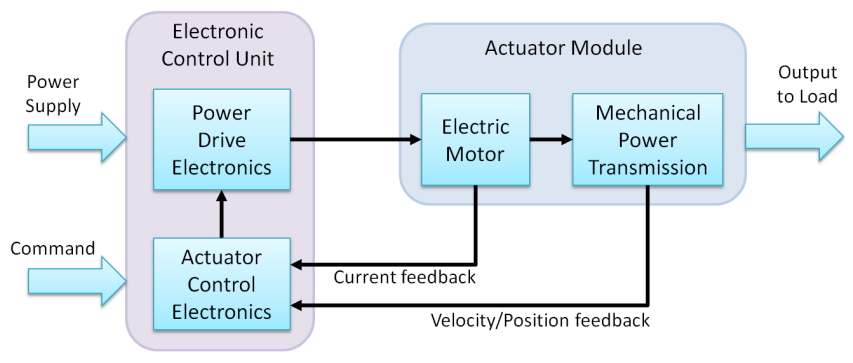

Figure 4: Schematic of a direct-drive linear EMA actuation system.

This work will concentrate on fault diagnosis of the EMA actuator module, centred on the MPT but also considering a simplified EM and load coupling models. The actuator model is proposed in the next section. 


\subsection{EMA Behavioural Model}

Since the EMA can be divided into different interconnected subsystems, the bond graph model can be constructed by an incremental approach, modelling each of the subsystems and then coupling these sub-models into the complete EMA model. The non-causal word bond graph model for the EMA, neglecting the control signals, is presented in fig. 5 .

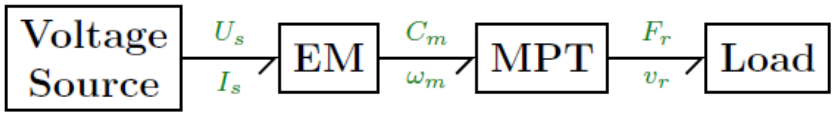

Figure 5: Word bond graph for a direct-drive EMA actuation system.

The EM performs electromechanical power conversion, being the key enabler for all kinds of PBW systems in the MEA concept. This fundamental device in currently implemented on EMAs using a high-performance permanent magnet synchronous motor (PMSM), which reduces the complexity of actuator control design [8]. A simplified model of the EM is based on a perfect DC motor, enabling perfect power transformation between the electrical and mechanical energy domains. On the bond graph formalism it is represented by a power gyrator (GY), which relates the flow variable of electric current $\left(I_{m}\right)$ on one port to the effort variable of electromagnetic torque $\left(C_{e m}\right)$ on the other port through the motor torque constant $\left(K_{m}\right)$. The major effects in the electric domain of the EM are the cooper loss, which can be defined as a voltage drop due to the winding resistance $\left(R_{w}\right)$ and modelled by a $\mathbf{R}$ element in bond graph, and the electrical storage effect due to the winding inductance $\left(L_{w}\right)$ and modelled by an I element. On the mechanical side of the EM the more important effects are the rotor inertia $\left(J_{m}\right)$ modelled by an I element and the mechanical friction $\left(R_{f m}\right)$ modelled by a $\mathbf{R}$ element

MPT is responsible to transform rotational into translational mechanical power, which is achieved using a roller-screw device where the torque $\left(C_{m}\right)$ and rotational velocity $\left(\omega_{m}\right)$ from the EM are converted into force $\left(F_{r}\right)$ and translational velocity $\left(v_{r}\right)$ of the actuator rod. A perfect roller-screw mechanism can be modelled by a bond graph power transformer element (TF) that relates the rotational power variables $\left(C_{m}, \omega_{m}\right)$ to the translational ones $\left(F_{n s}, v_{n s}\right)$ according to the transformation ratio $(2 p)$ of the screw pitch. Three additional significant effects are considered in the basic MPT model: roller-screw inertia, mechanical friction loss and compliance effects. For simplification the inertia is assumed to be concentrated on the translational side of the MPT, represented by the rod mass $\left(m_{r}\right)$ and modelled by a bond graph $\mathbf{I}$ element. The power loss is modelled as a viscous friction force $\left(F_{f r}\right)$, proportional to the operational velocity and the viscous coefficient $\left(R_{f r}\right)$, being represented by a bond graph $\mathbf{R}$ element. The compliance effect is due to the deformation of MPT mechanical components under stress and can be modelled by associated spring and damping effects. The linear spring effect is expressed by an elastic force $\left(F_{e}\right)$ proportional to the relative displacement and the compliance stiffness $\left(k_{c r}\right)$, while the damping force $\left(F_{d}\right)$, also a linear function, is proportional to the relative velocity and the damping coefficient $\left(R_{c r}\right)$. The spring and damping effects are respectively associated to a bond graph capacitance $(\mathbf{C})$ and resistance $(\mathbf{R})$.

The actuator load, composed of the flight control surface, is simply modelled as an equivalent translating mass $\left(m_{L}\right)$ represented by a bond graph $\mathbf{I}$ element. An effort source (Se) is also included to represent external disturbance aerodynamic forces $\left(F_{a e r}\right)$ applied over the control surface. In addition, a compliance effect similar to the one included on the MPT model is considered here, representing the spring and damping effects associated to the mechanical link between the control surface and the actuator rod.

The complete EMA behavioural bond graph model, which is presented in fig. 6 , is created by combining the EM, MPT, and load coupling sub-models. The model also includes four sensors, which are normally used in EMA systems: EM current $\left(i_{m}\right)$, EM rotational velocity $\left(\omega_{m}\right)$, MPT rod translational velocity $\left(v_{r}\right)$ and load translational velocity $\left(v_{L}\right)$.

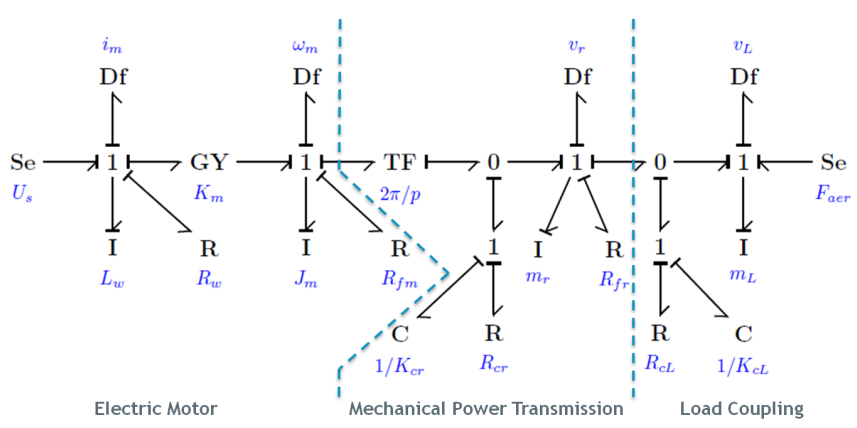

Figure 6: EMA behavioural bond graph model (integral causality).

The next step is to identify the most common failure modes that may affect the system and include these faults into the EMA behavioural model. Mechanical faults are credited to be the main source of concern for EMA systems. The rigorous conditions of aerospace applications lead to backlash, vibration and mechanical jamming on the roller-screw mechanism of the MPT, often caused by excessive loads, environmental factors, lubrication issues, and manufacturing defects. The second source of concern is the EM, where the high operational demands often leads to increased temperature within the motor housing, which may contribute to winding circuit faults. This work will consider the most probable failure modes [9] presented and described in the sequence:

Stator winding failure: higher temperatures will mostly affect the EM, leading to insulation deterioration on the stator winding and possibly resulting in short or open circuits which will directly affect the EM performance. The bond graph model already has a component representing the stator winding resistance $\left(R_{w}\right)$, what makes it easier to insert a short or open circuit fault by simply changing the resistance value.

Backlash: the excessive wear of the mechanical parts on the MPT, specially in the contact areas between the mov- 
ing parts will contribute to increased backlash, generating the so-called lost-motion and significantly affecting the dynamic performance of the actuator. When it exists, backlash can be considered as a compliance effect on the MPT model, an elastic force proportional to relative motion. Given that, a more realistic compliance model is necessary in order to represent not only the pure spring and damping effects but also the possible backlash and preload. Fu [8] proposes such a model, presented in fig. 7, which is incorporated in the bond graph EMA behavioural model.

Jamming: results in the complete blockage of the actuator movement and can be modelled on the MPT by increasing the mechanical friction parameters in order to force stiction. On the developed MPT bond graph model this can be simply implemented by increasing the viscous friction coefficient $\left(R_{f r}\right)$.

Mechanical disconnection: this fault is not related to EMA internal components but to the mechanical linkage between the actuator and the flight control surface, modelled here as a compliance effect on the load coupling. The mechanical disconnection, also called free-run, occurs in the case of a complete structural failure on the mechanical linkage, decoupling the actuator load.

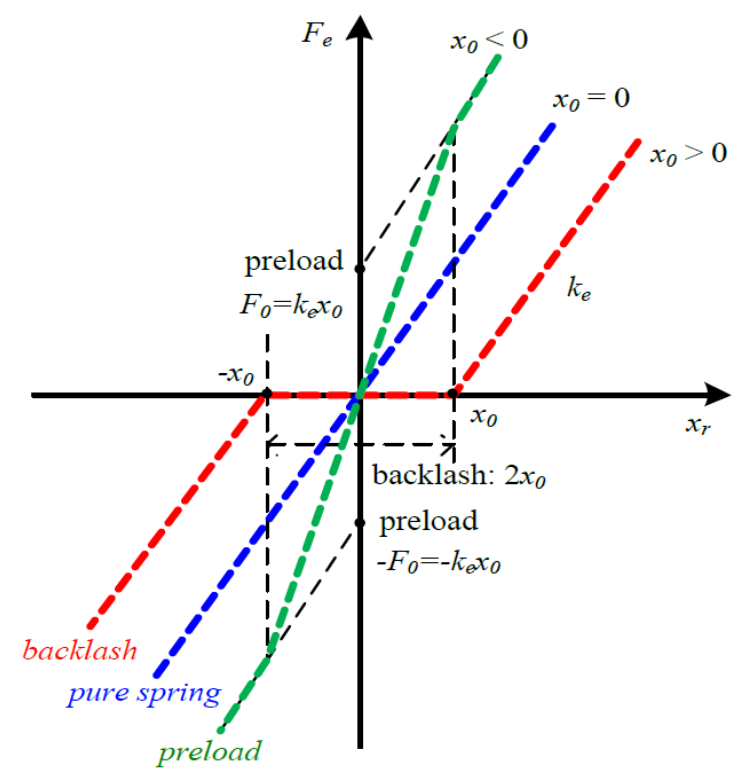

Figure 7: Compliance model with backlash and preload effects [8].

\subsection{EMA Diagnostic Bond Graph Model}

The DBG model is directly derived from the behavioural model performing the causality inversion of detectors, i.e. putting the model in preferred derivative causality and replacing the sensors by corresponding sources with their virtual residual sensors, or residual sinks. The diagnostic model will receive the signals coming from the behavioural model flow sensors and give the residuals in the virtual effort detectors
(De*). This approach enables the numerical residual evaluation without the need of symbolically deriving ARRs. It also guarantees that all virtual residual sensors will have different sets of causal paths, meaning that residuals obtained are always structurally independent, as described in Section 2.1.

The framework for FDI simulation involves coupling the behavioural and the diagnostic models. The resulting coupled model is presented in fig. 8. To simulate real instrumentation all sensor outputs from the behavioural model are contaminated with noise $\left(W_{1}\right.$ to $\left.W_{4}\right)$ before going to the diagnostic model. The noise model is based on white Gaussian noise with magnitude chosen at $1 \%$ of each signal measurement. Finally, the diagnostic model evaluates the signals coming from the behavioural model and gives the residuals (res 1 to $r e s_{4}$ ).

Once we have the residuals available from the DBG model, the next step for FDI is to obtain the fault signature matrix (FSM). This is accomplished through the analysis of the causal paths leading to each residual detector, resulting in the FSM presented in tab. 1, where the additional columns $D$ and $I$ indicates the detectability and isolability indexes, respectively. It is seen that faults in all of the components are detectable, i.e. at least one of the residuals is sensitive to that component, however, only fault in the rotational velocity sensor $\omega_{m}$ can be isolated, the other components do not have distinct fault signatures. Although most of the faults cannot be isolated to a single component, their signatures will indicate well defined segments of the system with a few possible faulty components.

Table 1: Fault Signature Matrix for the EMA system.

\begin{tabular}{lccccccc}
\hline Subsystem & Component & res $_{1}$ & res $_{2}$ & res $_{3}$ & res $_{4}$ & D & I \\
\hline EM electrical & $U_{s}$ & 1 & 0 & 0 & 0 & 1 & 0 \\
& $L_{w}$ & 1 & 0 & 0 & 0 & 1 & 0 \\
EM mechanical & $R_{w}$ & 1 & 0 & 0 & 0 & 1 & 0 \\
& $K_{m}$ & 1 & 1 & 0 & 0 & 1 & 0 \\
\multirow{3}{*}{ MPT roller-screw } & $J_{m}$ & 0 & 1 & 0 & 0 & 1 & 0 \\
& $R_{f m}$ & 0 & 1 & 0 & 0 & 1 & 0 \\
& $p$ & 0 & 1 & 1 & 0 & 1 & 0 \\
& $R_{c r}$ & 0 & 1 & 1 & 0 & 1 & 0 \\
Load coupling & $k_{c r}$ & 0 & 1 & 1 & 0 & 1 & 0 \\
\multirow{3}{*}{ Load } & $M_{r}$ & 0 & 0 & 1 & 0 & 1 & 0 \\
\multirow{3}{*}{ Sensors } & $R_{f r}$ & 0 & 0 & 1 & 0 & 1 & 0 \\
& $R_{c L}$ & 0 & 0 & 1 & 1 & 1 & 0 \\
& $k_{c L}$ & 0 & 0 & 1 & 1 & 1 & 0 \\
& $M_{L}$ & 0 & 0 & 0 & 1 & 1 & 0 \\
& $F_{a e r}$ & 0 & 0 & 0 & 1 & 1 & 0 \\
& $i_{m}$ & 1 & 1 & 0 & 0 & 1 & 0 \\
& $\omega_{m}$ & 1 & 1 & 1 & 0 & 1 & 1 \\
& $v_{r}$ & 0 & 1 & 1 & 0 & 1 & 0 \\
& $v_{L}$ & 0 & 0 & 1 & 1 & 1 & 0 \\
\hline
\end{tabular}

\section{Simulation and Results}

The behavioural and diagnostic bond graph models developed in Section 3 are implemented and simulated using the 20-sim software, by Controllab Products [10]. In order to evaluate the aspects of the main dynamic effects of the proposed model, the EMA is simulated with real system parameters, gathered from industry product specifications and from the literature. These parameters are listed in tab. 2. 


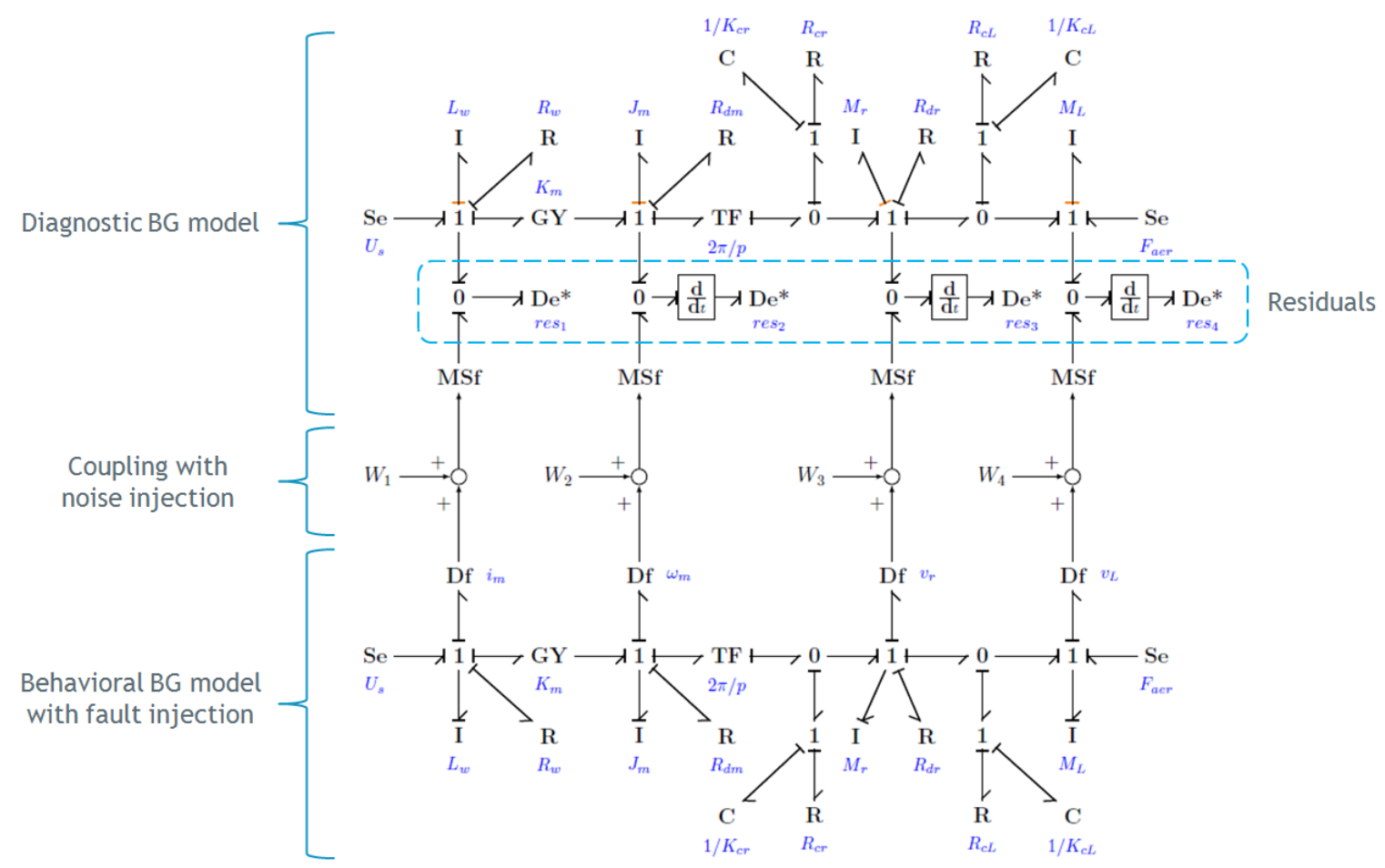

Figure 8: Coupling of EMA behavioural and DBG models.

Table 2: EMA model parameters.

\begin{tabular}{crll}
\hline Parameter & \multicolumn{1}{c}{ Value } & \multicolumn{1}{c}{ Unity } & \multicolumn{1}{c}{ Description } \\
\hline$J_{m}$ & 0.001279 & $\mathrm{~kg} \mathrm{~m}^{2} \mathrm{rad}^{-1}$ & EM rotor inertia \\
$K_{c L}$ & $3 \times 10^{8}$ & $\mathrm{Nm}^{-1}$ & Load compliance stiffness \\
$K_{c r}$ & $3 \times 10^{8}$ & $\mathrm{~N} \mathrm{~m}^{-1}$ & Roller-Screw compliance stiffness \\
$L_{w}$ & 3 & $\mathrm{mH}$ & EM stator winding inductance \\
$M_{L}$ & 600 & $\mathrm{~kg}$ & Load reflected mass \\
$M_{r}$ & 1 & $\mathrm{~kg}$ & Roller-screw rod mass \\
$p$ & 2.54 & $\mathrm{~mm}$ & Lead of roller-screw \\
$R_{c L}$ & $1 \times 10^{4}$ & $\mathrm{~N} \mathrm{~s} \mathrm{~m}^{-1}$ & Load compliance damping \\
$R_{c r}$ & $1 \times 10^{4}$ & $\mathrm{Ns} \mathrm{m}^{-1}$ & Roller-screw compliance damping \\
$R_{f m}$ & $1 \times 10^{-3}$ & $\mathrm{Ns} \mathrm{m}^{-1}$ & EM friction coefficient \\
$R_{f r}$ & $1 \times 10^{4}$ & $\mathrm{Ns} \mathrm{m}^{-1}$ & Roller-screw friction coefficient \\
$K_{m}$ & 0.46 & $\mathrm{Nm} \mathrm{A}^{-1}$ & EM torque constant \\
$R_{w}$ & 1.5 & $\Omega$ & EM stator winding resistance \\
$U_{s}$ & 115 & $\mathrm{~V}$ & Bus voltage \\
\hline
\end{tabular}

Source: $[8,11,12]$.

First, the behavioural model for the EMA is implemented and simulated, validating the system open-loop response and its behaviour in all modelled failure modes: backlash, jamming, mechanical disconnection, stator open-circuit and stator short-circuit. The diagnostic model is then implemented and coupled with the behavioural model, following the FDI framework presented in fig. 8. The coupled model is simulated in all failure modes, generating the residuals, which are evaluated for fault detection and isolation.

For normal operation, the system is simulated in open-loop, with a $115 \mathrm{~V}$ step input on the voltage source $\left(U_{S}\right)$ and a step aerodynamic force disturbance $\left(F_{\text {aer }}\right)$ of $3 \mathrm{kN}$. Figure 9 gives the EMA simulated open-loop response for normal operation, which is as expected. The load displacement velocity $\left(v_{L}\right)$ stabilizes at $0.1 \mathrm{~m} \mathrm{~s}^{-1}$ after a fast transient oscillation. It is also observed that the stiffness and damping compliance effects of the MPT and load coupling contribute significantly to increasing the load velocity oscillations. The electric motor stator winding current $\left(i_{m}\right)$ presents a transient peak of about $60 \mathrm{~A}$, which shall be controlled in a closed-loop implementation. Neither of the residuals will exceed the experimental thresholds, showed in fig. 10, indicating the system is operating without failures.

The EMA response to failure is evaluated by injecting the 

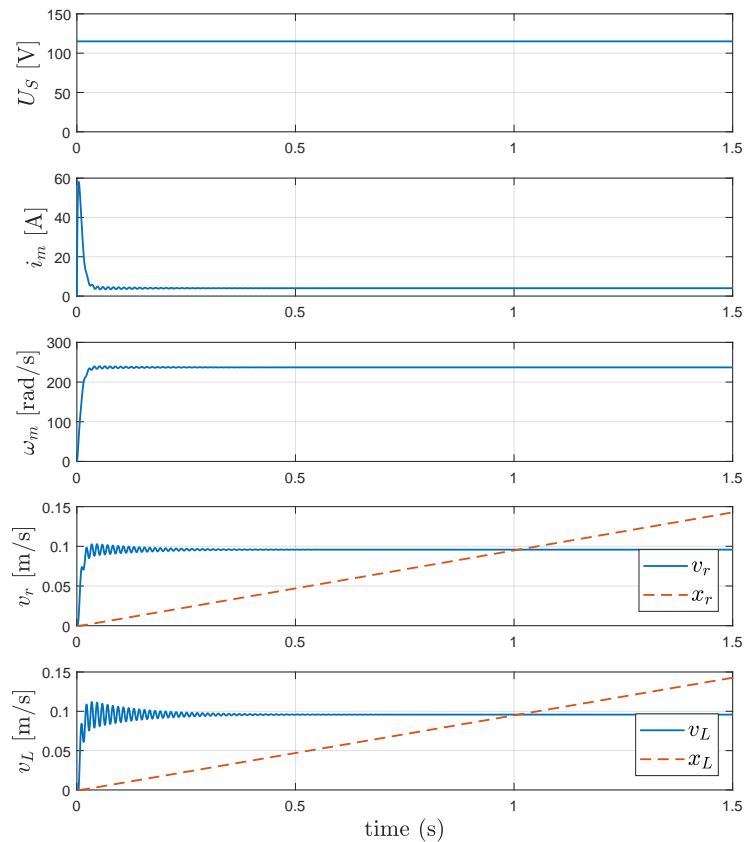

Figure 9: Simulation results for the EMA behavioural model in normal operation.
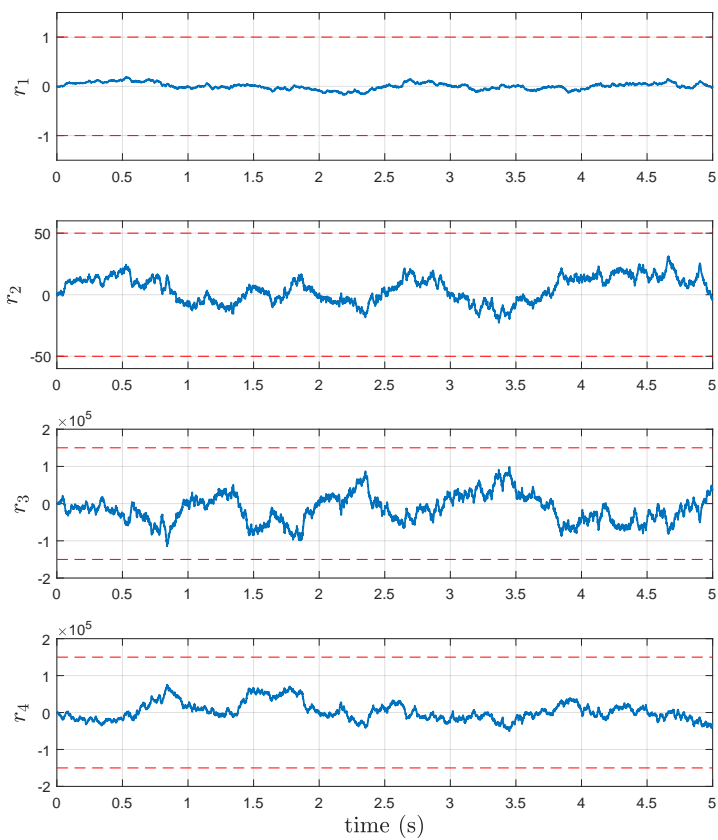

Figure 10: Residuals for the simulation of EMA coupled models in normal operation. Residual thresholds are displayed in red dashed lines.

faults modelled in Section 3.1. The model is simulated with the same parameters as before and the faults are injected, one at a time, as a step input always at $1 \mathrm{~s}$ of simulation.
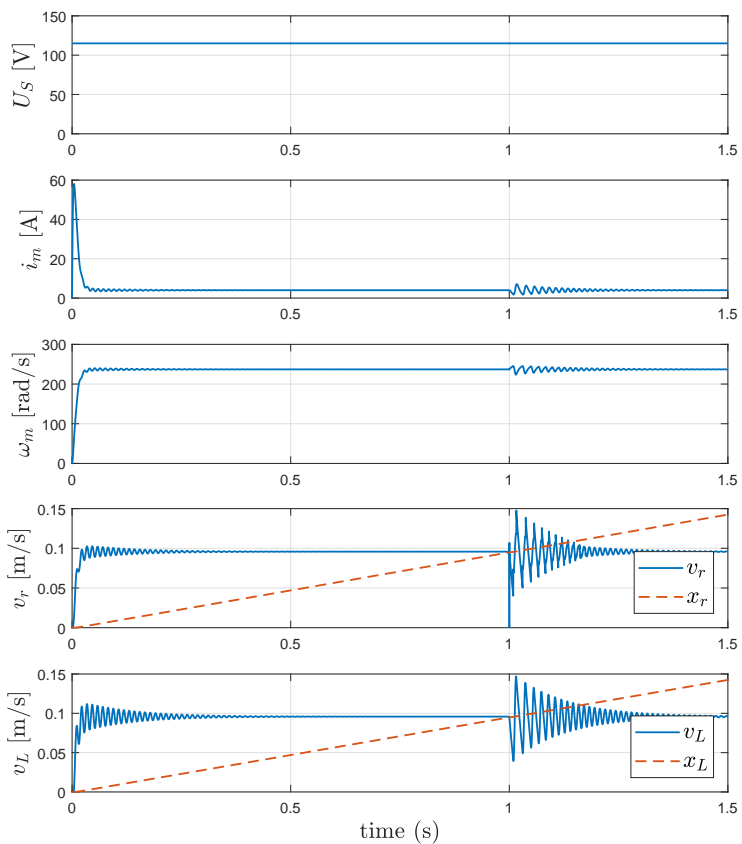

Figure 11: Simulation results for the EMA behavioural model with $0.3 \mathrm{~mm}$ backlash.
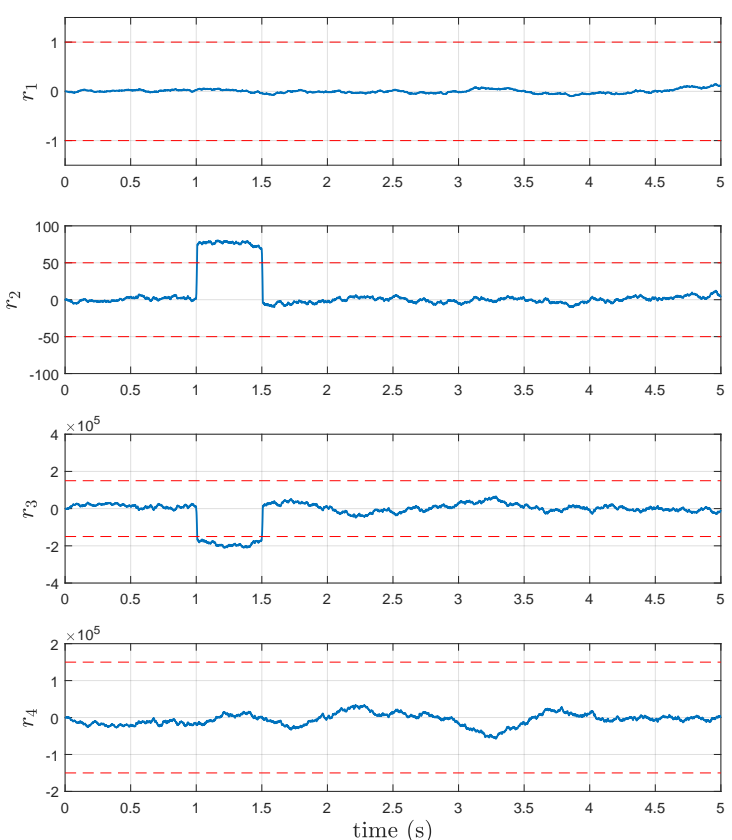

Figure 12: Residuals for the simulation of EMA coupled models with $0.3 \mathrm{~mm}$ backlash. Residual thresholds are displayed in red dashed lines.

As an example, the simulation results for the backlash fault are presented in fig. 11. The compliance effects on the mechanical power transmission are implemented in 20-sim ac- 
Table 3: FDI results summary.

\begin{tabular}{cccc}
\hline Fault & Fault Injection & Coherence Vector & Fault Candidates \\
\hline Normal operation & - & {$[0,0,0,0]$} & - \\
Open-circuit & $R_{w}$ & {$[1,0,0,0]$} & $U_{s}, L_{w}, R_{w}$ \\
Short-circuit & $R_{w}$ & {$[1,0,0,0]$} & $U_{s}, L_{w}, R_{w}$ \\
Backlash & $R_{c r}, K_{c r}$ & {$[0,1,1,0]$} & $p, R_{c r}, K_{c r}, v_{r}$ \\
Jamming & $R_{f r}$ & {$[0,0,1,0]$} & $M_{r}, R_{f r}$ \\
Mechanical disconnection & $R_{c L}, K_{c L}$ & {$[0,0,1,1]$} & $R_{c L}, K_{c L}, v_{L}$ \\
\hline
\end{tabular}

cording to the backlash model given in fig. 7, the results show the actuator response for a $0.3 \mathrm{~mm}$ backlash applied at $1 \mathrm{~s}$. Backlash will occur due to excessive wear in the contact surfaces of the roller-screw mechanical system, creating a lostmotion effect and introducing oscillations that will propagate throughout the entire system, affecting the EMA dynamic performance.

The residuals evaluation, presented in fig. 12 show that the defined threshold is crossed for residuals $r_{2}$ and $r_{3}$. Giving the coherence vector $C=[0,1,1,0]$, which according to the FSM, means there is a faulty component in the MPT mechanism (parameters $p, R_{c r}$ or $K_{c r}$ ) or in the actuator velocity sensor $\left(v_{r}\right)$, which represents a correct diagnostic.

All failure modes were simulated and the DBG residuals evaluation pointed to the correct diagnostics, according to the fault signature matrix. Table 3 presents a summary of the simulation results.

\section{Discussion}

The recent progresses in PBW technology is driving the EMA as the next generation of actuation systems, already replacing conventional hydraulic actuators in commercial and military aerospace applications. However, their use in safety critical systems, such as primary flight controls, still faces some obstacles until it proves to be reliable and to behave safely, especially in fault scenarios. One of the research topics that can help on the evolution and acceptance of EMA in future aircraft architecture is the development of condition monitoring and diagnostic systems, such as the proposed FDI methodology.

The developed bond graph models allowed the simulation of an EMA system in different faulty conditions, providing an easy to implement diagnostic system which can be really helpful in the early diagnosis of EMA faults, improving its acceptance in aerospace applications. These models can be used for evaluation during the early process of EMA design, without the need of any real prototypes and expensive test sets.

The models were implemented in the 20-sim simulation environment, as presented in Section 4. First, the open-loop behavioural model was simulated to verify its response. The use of a behavioural model as a tool for fault simulation brings the advantage of introducing any kinds of faults without the risks associated with the real process. The DBG model was then coupled with the behavioural model, generating numeri- cal residuals which were processed and evaluated for the definition of experimental thresholds. The diagnostic result was correct to all simulated failure modes, confirming the feasibility of the DBG method proposed in the literature [6] and its application to EMA development.

Opportunities derived from this work, aiming the further development of the proposed method are listed below, as suggestions for future research topics:

1. Refinement of the EMA model, including the power drive electronics and actuator control electronics models, with its respective failure modes;

2. Implementation of the complete EMA controller and analysis of the system closed-loop response, specially on the impacts of the control loop over the response to different failure modes;

3. Analysis of multiple-fault scenarios, applying multiplefault isolation techniques based on parameter estimation by Gauss-Newton least squares output error minimization $[13,14]$;

4. Development of Bicausal Bond Graph (BBG) models for analysis and improvement of sensor placement and residuals generation [15];

5. Use of uncertain bond graph models for robust FDI residuals generation, and sensitivity analysis of the residuals in the presence of parameter uncertainties [16];

6. Study of reconfigurable Fault Tolerant Control (FTC) techniques for fault accommodation / passivation [17];

7. DBG online implementation experiment, the outputs of the behavioural model are to be replaced by measured outputs from the real EMA system.

\section{Conclusions}

This paper presented a bond graph model-based approach to FDI, showing its application to an electromechanical actuator, relevant to MEA actuation systems. The bond graph modelling formalism is used as the base of the DBG FDI method being proposed. The DBG makes use of virtual residual detectors, also called residual sinks, enhancing the availability of information for the diagnostic system by generating all the structurally independent residuals in numerical form, as the numerical evaluation of ARRs. This is important in situations 
where symbolic ARRs cannot be obtained due to the presence of algebraic loops in the system bond graph model. The DBG is also used for the construction of a FSM by analysis of the causal paths leading to the residuals.

The DBG approach was proven as a powerful tool, suitable for the implementation of FDI on complex multidisciplinary systems, such as the EMA. It is concluded that there are significant advantages in using the bond graph formalism, when compared to other modelling methods, as a tool for developing model-based quantitative FDI, confirming its capacity on the diagnostic of EMA faults. Still, the FDI approach proposed here is not restricted to this use, being applicable on the diagnostic of any complex interdisciplinary system such as those commonly found on the aerospace industry.

\section{References}

[1] Guan Qiao, Geng Liu, Zhenghong Shi, Yawen Wang, Shangjun Ma, and Teik C Lim. A review of electromechanical actuators for More/All Electric aircraft systems. Proceedings of the Institution of Mechanical Engineers, Part C: Journal of Mechanical Engineering Science, 2017.

[2] Michel Todeschi and Laura Baxerres. Health monitoring for the flight control EMAs. IFAC-PapersOnLine, 28(21):186-193, 2015.

[3] Jean Charles Maré. Aerospace actuators 2 : signal-bywire and power-by-wire. John Wiley \& Sons, 2017.

[4] SAE. ARP4761, Guidelines and Methods for Conducting the Safety Assessment Process on Civil Airborne Systems and Equipment. SAE International, 1996.

[5] Venkat Venkatasubramanian, Raghunathan Rengaswamy, Kewen Yin, and Surya N. Kavuri. A review of process fault detection and diagnosis: Part I: Quantitative model-based methods. Computers and Chemical Engineering, 27(3):293-311, 2003.

[6] Arun K. Samantaray and Belkacem Ould Bouamama. Model-based process supervision : a bond graph approach. Springer, London, 2008.

[7] Wolfgang Borutzky. Residual Bond Graph Sinks for Numerical Evaluation of Analytical Redundancy Relations in Model Based Single Fault Detection and Isolation. Proc. 20th European Conference on Modelling and Simulation (ECMS'06), 5(Cd):166-172, 2006.

[8] Jian Fu, Jean Charles Maré, and Yongling Fu. Incremental modeling and simulation of mechanical power transmission for more electric aircraft flight control electromechanical actuation system application. ASME International Mechanical Engineering Congress and Exposition, Proceedings (IMECE), 1, 2016.

[9] Edward Balaban, Abhinav Saxena, Prasun Bansal, Kai F. Goebel, Paul Stoelting, and Simon Curran. A diagnostic approach for electro-mechanical actuators in aerospace systems. IEEE Aerospace Conference Proceedings, pages 1-13, 2009.

[10] C. Kleijn, M. A. Groothuis, and H. G. Differ. 20-sim 4.6 Reference Manual. Controllab Products B.V., Enschede, 2017.

[11] EXLAR. Product Specifications. GSX Series Integrated Motor Actuator, 2018.

[12] Lijian Wang and Jean Charles Maré. A force equalization controller for active/active redundant actuation system involving servo-hydraulic and electro-mechanical technologies. Proceedings of the Institution of Mechanical Engineers, Part G: Journal of Aerospace Engineering, 228(10):1768-1787, 2014.

[13] Arun K. Samantaray and Sanjoy K. Ghoshal. Sensitivity bond graph approach to multiple fault isolation through parameter estimation. Proceedings of the Institution of Mechanical Engineers. Part I: Journal of Systems and Control Engineering, 221(4):577-587, 2007.

[14] Wolfgang Borutzky. Bond graph methodology: Development and analysis of multidisciplinary dynamic system models. Springer, London, 2010.

[15] Arun K. Samantaray and Sanjoy K. Ghoshal. Bicausal bond graphs for supervision: From fault detection and isolation to fault accommodation. Journal of the Franklin Institute, 345(1):1-28, 2008.

[16] Mohand A. Djeziri, Belkacem Ould Bouamama, and Rochdi Merzouki. Modelling and robust FDI of steam generator using uncertain bond graph model. Journal of Process Control, 19(1):149-162, 2009.

[17] Youmin Zhang and Jin Jiang. Bibliographical review on reconfigurable fault-tolerant control systems. Annual Reviews in Control, 32(2):229-252, 2008. 